\title{
Sample size and the detection of means: A signal detection account
}

\author{
Richard B. ANDERSON ANd Michael E. DOHERTY \\ Bowling Green State University, Bowling Green, Ohio
}

\begin{abstract}
Using statistical theory as a basis, Kareev (e.g., 1995) claimed that people's ability to correctly infer the existence of a population correlation should be greater for small than for large samples. Simulations by R. B. Anderson, Doherty, Berg, and Friedrich (2005) identified conditions favoring small samples but could not determine whether such an advantage was due to sampling skew, variance, or central tendency displacement. In the present study, we investigated theoretical effects of sample size $(n)$ on the detection of population means under circumstances in which sampling variance is unconfounded with skew or central tendency displacement. The results demonstrate an extremely limited, criterion-specific, small-sample advantage that was attributable to $n$-related sampling variance and that occurred only with highly conservative, suboptimal criterion placement.
\end{abstract}

Numerous theorists have argued that a crucial first step in investigating the psychology of an organism is to gain insight into the environment in which the organism has evolved and to which it has adapted. Brunswik (1956, p. 119) used strong phraseology in describing the analysis of "textural ecology as a propaedeutic to functional psychology." He had previously exemplified this prescription with his work on the ecological validity of the Gestalt principle of proximity (Brunswik \& Kamiya, 1953). Brunswik's call for the study of the ecology has been characterized as "psychology without a subject," emphasizing the importance of studying the environment (see, e.g., Doherty, 2001). An example of this notion was introduced by Simon (1969), who described the complex behavior of an ant on a beach as a "reflection of the complexity of the environment in which it finds itself" (p. 24). Further calls for the necessity of modeling the environment have been made by J. R. Anderson (1990), Shepard (1990), and Gigerenzer and Todd (1999). As Shepard (1990, p. 213) succinctly put it, "what we are beginning to discern about the mind looks very much like a reflection of the world."

\section{QUANTIFYING DECISION ACCURACY}

It is well-known that for any given level of tolerance $(\alpha)$ for false positives, the power for detecting an effect increases monotonically with sample size. Thus, it is clear that large samples are better than small ones when decision accuracy is measured according to the prescriptions of null hypothesis testing. Within this prescriptive framework, it is assumed that there is a default and, thus, privileged hypothesis about some aspect of the environment. Moreover, this "null" hypothesis is rejected when $\alpha$, the probability of incorrectly rejecting the null (which can be conceptualized as a false alarm rate), is less than or equal to some agreed-upon standard. The logic of null hypothesis testing typically assumes that the decision maker should be unwilling to accept a greater-than- $\alpha$ error rate, even though the result would be to substantially increase statistical power-that is, to increase the probability of rejecting the null hypothesis given that the hypothesis is false.

In contrast to null hypothesis testing, signal detection theory (see Macmillan \& Creelman, 1991) assumes that data have been sampled from either a signal population or a noise population. It is also assumed that each datum is characterized by a particular value on an evidence scale and that the level of the evidence variable is diagnostic as to whether the datum was drawn from a signal population or a noise population. The decision maker's task is to pick a particular critical value on the evidence scale. When the evidence variable exceeds the critical value, the stimulus is judged to be a signal; otherwise, it is judged to be noise.

For example, in research on recognition memory (for a review, see Yonelinas, 2002), subjects are typically presented first with a study list and then with a recognition list that contains both studied and nonstudied items. The task is to respond "yes" or "no" to each recognition item to indicate whether that item was or was not studied, respectively. In a signal detection analysis of the results, it is assumed that each recognition item possesses a particular level of familiarity (the evidence variable) and that the level of familiarity tends to be higher for studied than for nonstudied items. In this example, the studied items make up the signal population; the nonstudied items make up the noise population. It is assumed that subjects attempt to maximize decision accuracy by adopting a particular crite- 
rion (in this case, a familiarity criterion) that appropriately discriminates signals from noise: The subject says "yes" if the value of the evidence variable exceeds the criterion and "no" otherwise. Given sufficient knowledge of the shapes of the distributions (i.e., the item frequencies for each population as a function of the evidence variable), the decision maker can set the criterion to an optimal level (i.e., one that maximizes performance accuracy) so that when and only when the criterion is exceeded, the item is objectively more likely to be a signal than noise. To the extent that knowledge of the distributions is incomplete, criterion placement is subject to error. If there is no knowledge of the distributions, the criterion must be set arbitrarily.

Signal detection performance can be measured in various ways (see Stanislaw \& Todorov, 1999, for a review). One way is to calculate the hit rate ( $H$-proportion of signals judged to be signals) and the false alarm rate ( $F$ - proportion of noise trials judged to be signals); use these two rates to reconstruct the internal, psychological distributions of signals and noises; and then measure performance as the degree of separation $\left(d^{\prime}\right)$ between the two internal distributions. The separation of the distributions can also be assessed by encouraging the subject to repeat the task while varying the criterion levels across the repetitions. The resulting $H \mathrm{~s}$ and $F \mathrm{~s}$ are then plotted as a curve showing $H$ as a function of $F$, and the area under the curve is taken as a measure of the separation between the signal and noise distributions.

Whereas the computation of $d^{\prime}$ and the area under an ROC curve both entail an aggregation of performance across all criterion levels, one may also ask what level of accuracy the decision maker obtains when he or she places the criterion at a particular (and perhaps arbitrary) level. In such a case, criterion-specific accuracy can be measured as net gain - that is, $H-F .^{1}$ Note that if the values of the hits and false alarms were asymmetrical-for example, if the cost of a false alarm outweighed the benefit of a hit - then net gain would more appropriately be computed as the weighted $H$ minus the weighted $F$. However, because the present simulations use equal-valued hits and false alarms, net gain simplifies to $H-F$.

\section{SAMPLE SIZE AND THE STATISTICAL ECOLOGY}

\section{Sampling Distributions of $\boldsymbol{r}$ and Fisher's $\boldsymbol{z}$}

Correlation detection-that is, the ability to judge whether or not two variables are related-is an important aspect of cognition. For example, it is beneficial to be able to tell whether or not there is a correlation between the price paid for a particular drug and its effectiveness, between the degree of one's effort and the likelihood of success, or between the positions of the stars and one's good fortune. Such correlations must often be inferred from a limited sample of data.

Recent investigations of correlation judgment suggest that there are particular ecological conditions favoring small rather than large samples. This line of inquiry was initiated by a provocative article by Kareev (1995) that flew in the face of conventional wisdom. Kareev (1995) noted that for a nonzero population correlation $(\rho)$, the sampling distribution of Pearson's correlation coefficient $(r)$ is skewed and that the skewness increases as sample size $(n)$ decreases. Thus, the likelihood that $r$ will exceed some critical $r$, leading to the correct decision that $\rho>0$, increases as $n$ decreases. Figure 1 illustrates this phenomenon.

Starting from the analysis of the statistical ecology in which inferences about relationships are drawn, Kareev (1995, p. 267) asserted that ". . . the fact that working memory capacity is limited increases the chances of detecting a correlation," but later acknowledged that what he termed the narrow window advantage may be partly offset by the greater likelihood of false alarms (Kareev, 2000).

In subsequent simulation research, Juslin and Olsson (2005) and R. B. Anderson, Doherty, Berg, and Friedrich (2005) confirmed that false alarms can offset what would otherwise be a small-sample advantage. However, R. B. Anderson et al. showed that when the to-be-detected correlation is moderate and the decision criterion is conservative rather than liberal (i.e., when the criterion was set to keep the $F$ low), decision accuracy can in fact be greater for small than for large samples. Empirical evidence for the small-sample advantage includes data suggesting that

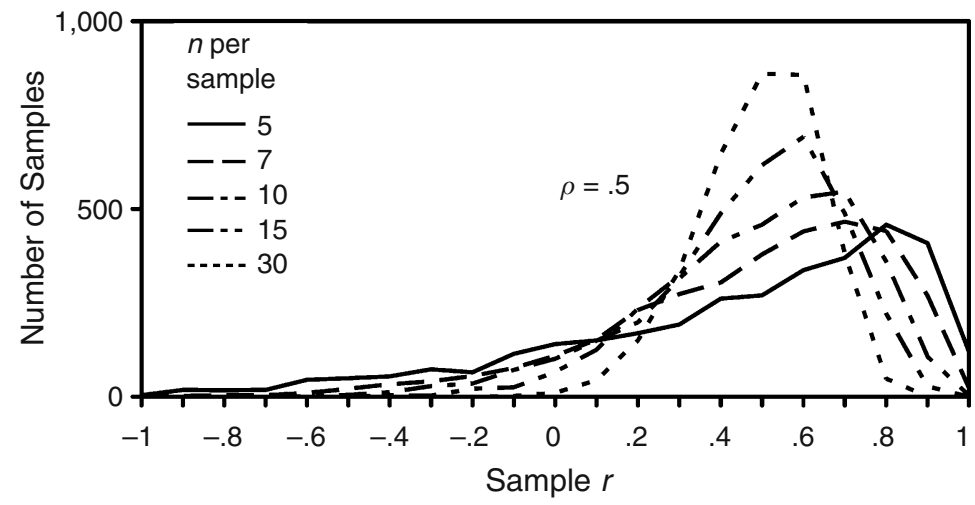

Figure 1. Empirical sampling distributions of the Pearson correlation coefficient for $n=5,7,10,15$, and 30 , for a population correlation $(\rho)$ of .5. Adapted from R. B. Anderson et al. (2005). 
a small working memory capacity can amplify the magnitudes of objective correlations (Kareev, Lieberman, \& Lev, 1997) and that, in consistency with R. B. Anderson et al., the performance advantage for small samples is present when the decision criterion is conservative rather than liberal (Fiedler \& Kareev, 2006).

Whereas the original impetus for this research was the recognition of skew in the sampling distribution, R. B. Anderson et al. (2005) suggested that the limited advantage for small samples may result not from skewness per se but from differential variance, central tendency displacement, or a combination thereof. This conclusion was based on the demonstration that the small-sample advantage is not limited to sampling distributions of $r$, but also occurs with normally distributed Fisher's $z$ coefficients. Thus, as $n$ decreases, not only does the Fisher's $z$ distribution become more variable, but the mean of the Fisher's $z$ distribution becomes progressively more extreme than the population parameter, as is illustrated in Figure 2.

Although the foregoing results helped to identify several possible causes for the small-sample advantage, it remains the case that sampling skew is mathematically confounded with sampling variance (for distributions of $r$ ) and with central tendency displacement (for distributions of the Fisher's $z$ transformation of $r$ ). Consequently, R. B. Anderson et al. (2005) could not draw a firm conclusion as to whether variance, skew, displacement, or a combination of these factors was a sufficient condition for producing a small-sample advantage.

\section{The Detection of Means}

Psychological significance. A variety of situations illustrates the importance of mean detection. For example, a person may suspect that he or she has a fever and may consequently use a thermometer to test that hypothesis. Given that the thermometer may be lacking in reliability, the person may take several measurements prior to drawing a conclusion about the presence or absence of fever. In this situation, one may construe the decision maker's task to involve calculating the mean of the measurements, then using that mean as a criterion for deciding whether the sample of measurements has been drawn from a signal distribution or from a noise distribution - that is, from a distribution of samples that have a mean of 98.6 or from one with a mean of, say, 99.0 , respectively. ${ }^{2}$ Note that such a procedure can be followed even if only one measurement is taken; in such a case, the sample mean would equal the value of the single measurement. It should be noted that a signal detection strategy requires the decision maker to have two possible distributions in mind. This is because the goal is to set the criterion so as to maximize net gain (or some other suitable index of decision accuracy). In addition, a reasonable guess as to the mean of the signal distribution must be based on prior experience or on some objective data relevant to the body temperatures of healthy and ill people. Therefore, a single sample does not provide sufficient information to support optimal placement of the criterion. Moreover, even with multiple samples, the decision maker would need additional information to establish appropriate value weightings for the hits and false alarms.

Other examples of mean detection methods include using a score on a neuropsychiatric examination to decide whether or not a patient has suffered a brain injury, and using the average amount of beer consumed by a group of friends as a cue to decide whether or not the group belongs to a fraternity.

Relevance to correlation detection. In R. B. Anderson et al.'s (2005) simulation of correlation detection, as $n$ per sample decreased, the sampling distribution for the signal population simultaneously became more variable and more skewed (see Figure 1 of the present article). Thus, as was noted above, there was an intrinsic mathematical confound between sampling variance and skew (or between sampling variance and distribution displacement when the Fisher's $z$ transform was applied; see Figure 2), which prevented the authors from drawing conclusions about the sufficient conditions for criterion-specific small-sample advantages in correlation detection.

However, absent floor or ceiling effects, there is no such confound regarding the detection of means. The distribu-

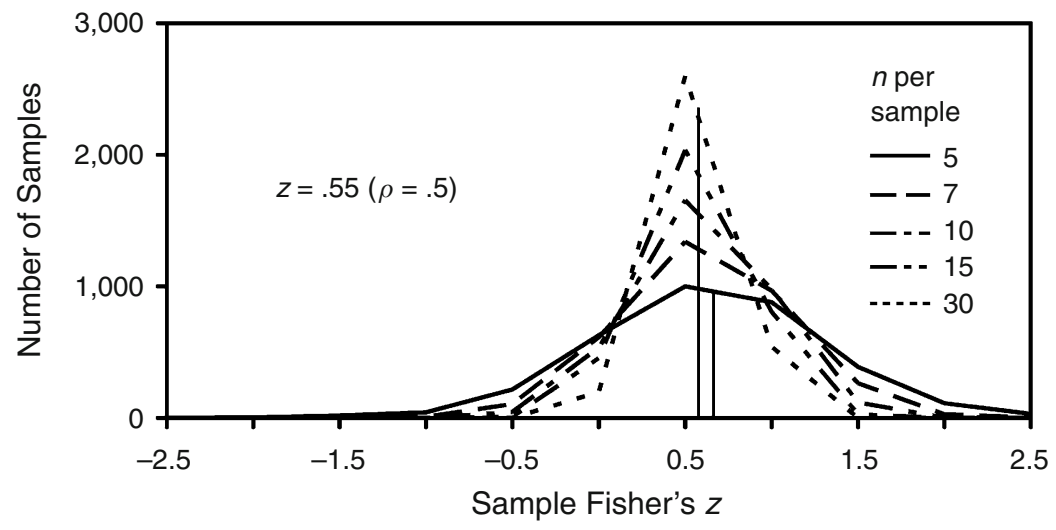

Figure 2. Empirical sampling distributions of Fisher's $\boldsymbol{z}$ for $\boldsymbol{n}=\mathbf{5}, \mathbf{7}, \mathbf{1 0}, \mathbf{1 5}$, and 30 , for population Fisher's $z$ of 0.55 (the corresponding $\rho$ is .5 ). Left and right vertical lines indicate the means of the distributions $n=5$ and $n=30$, respectively. Adapted from R. B. Anderson et al. (2005). 
tion of $\bar{X}$ remains symmetrical (unlike the $r$ distribution) and centered at the value of the population parameter (unlike the Fisher's $z$ distribution) irrespective of $n$. Thus, a major benefit of studying mean detection is that it provides a way to study detection under circumstances in which sampling variance is unconfounded with sampling skew or with displacement of the sampling distribution. Indeed, this characteristic of the sampling distribution of $\bar{X}$ is at the heart of the rationale for the present study, quite apart from any direct psychological significance of mean detection (although the case for direct psychological significance has been made, above).

\section{Predictions}

Under the assumption that sampling variance is, in fact, a sufficient condition for a small-sample advantage, it is predicted that a signal detection analysis of the sampling distribution of $\bar{X}$ will demonstrate a limited, criterionspecific advantage for small samples. Specifically, when the decision criterion is liberal, net gain should be greater for larger than for smaller samples, but when the criterion is conservative, decision accuracy should be greater for smaller than for larger samples. Such a result would generalize the findings for the detection of contingency to the detection of central tendency, and would strongly imply that sampling variance is a sufficient condition for producing a small-sample advantage in correlation detection.

\section{METHOD}

The contingency research, described previously (see, e.g., R. B. Anderson et al., 2005), relied on computer simulations to construct empirical sampling distributions. This is because the shapes of the sampling distributions of $r$ vary radically with $n$. It should be noted that conversion of $r$ to Fisher's $z$ did not obviate the need for simulation because the Fisher's $z$ approximation to normality becomes progressively poorer with smaller values of $n$ (David, 1954). However, given a normally distributed population, sampling distributions of $\bar{X}$ are normal irrespective of $n$. Therefore, the present article will employ theoretical rather than empirical, simulation-based sampling distributions.

The computations were conducted within the framework of signal detection theory and modeled a process wherein a hypothetical decision maker encounters a sample of $1,5,10,20$, or 40 values drawn from normally distributed noise values $(\mu=0, \sigma=1)$ or signal values $(\mu>0, \sigma=1)$. Therefore, for the noise populations five normal curves were computed to represent five distributions of sample means $(\bar{X} \mathrm{~s})$, with $n$ per sample equal to $1,5,10,20$, and 30 . Each of the curves was constructed with $\mu \bar{X}=0$. As per the central limit theorem, each curve had $\sigma \bar{X}=1 / \sqrt{n}$, where $n$ denotes the number of observations per sample. Curves for the signal distributions were constructed similarly to those for the noise distributions, except that the signal distribution means were manipulated across six levels: $0.5,1.0,1.5,2.0,2.5$, and 3.0. The decision criterion $(c)$ varied from 0.0 to 5.0 in steps of 0.25 . When $\bar{X}$ exceeded $c$, it was counted as a hit if it was drawn from a signal population or as a false alarm if it was drawn from a noise population.

The $H \mathrm{~s}$ and $F \mathrm{~s}$ were calculated from the theoretical sampling distributions using an algorithm (SPSS Version 11.0) for computing the cumulative probability $(\Pi)$, given $c$ (for a normal distribution with $\mu$ and $\sigma$ as parameters). Thus, for each level of signal $\mu$ and for each level of $c$, the $H$ was equal to $1-\Pi$ for the signal distribution, and the $F$ was equal to $1-\Pi$ for the noise distribution. Figure 3 illustrates $H \mathrm{~s}$ and $F$ s for signal $\mu=1.5, \mu=0$, and $c=1.75$ across five levels of $n$.

\section{RESULTS}

\section{Criterion-Independent Accuracy: ROC Curves}

ROC curves were constructed to assess the effect of $n$ on performance using an accuracy index that aggregates across criteria. Thus, the ROC curves measured criterionindependent performance. Each point in Figure 4 shows $\mathrm{H}$ and $\mathrm{F}$ for a given level of $c$; the area under each curve indicates the criterion-independent discriminability (often referred to as sensitivity) of signal from noise populations. Consistent with the findings of R. B. Anderson et al. (2005) for correlation detection, discriminability tended to increase with $n$, thus demonstrating an advantage in mean detection for large samples, not for small ones. This data pattern is similar to the discriminability results reported in R. B. Anderson et al.

\section{Criterion-Specific Accuracy}

Net gain. Whereas the ROC curves provided a general measure of accuracy, aggregated across criteria, accuracy was also measured in a criterion-specific fashion. One such criterion-specific measure is net gain, defined earlier as the difference between $H$ and $F$ (when hits and false alarms are symmetrically valued) for each level of the decision criterion. Figures 5 and 6 show the $H$ s (top panel), $F$ s (middle panel), and $H-F$ (bottom panel). For $\mu=.5$ (left panel in Figure 5), when criterion $c$ was zero, $F$ (shown in the middle panel) was 0.5 irrespective of $n$, but $H$ (top panel) increased with $n$. Consequently, $H-F$ increased with $n$. Moreover, as $c$ rose above zero, $H$ decreased faster for large than for small values of $n$, and because the differential decrease was even more pronounced for false alarms, $H-F$ increased and did so more dramatically for large than for small samples. However, as $c$ increased further, there was a crossover in the pattern of $\mathrm{Hs}$ so that small samples produced higher $H$ s than did large samples; the crossover occurred at the mean of the signal distribution. As $c$ increased yet further, it became increasingly probable that $\bar{X}$ would never exceed $c$, thereby causing $H, F$, and $H-F$ to fall to zero.

The same general data pattern was present for all levels of signal $\mu$, with the crossover point (for $H$ ) increasing as $\mu$ increased. Thus, consistent with the predictions, $H-F$ was greater for small than for large samples when the level of the decision criterion was relatively high.

Posterior probability. Another measure of criterionspecific accuracy is posterior probability (see Juslin \& Olsson, 2005). The posterior probability of a hit $\left(H_{\mathrm{p}}\right.$, where the subscript refers to posterior probability) is equal to $H /(H+F)$; the related measure, posterior probability of a miss $\left(M_{\mathrm{p}}\right)$ is equal to $M /(M+V)$, where $M$ is the miss rate (equal to $1-H$ ) and $V$ is the correct rejection rate (equal to $1-F)$. (Note that, as with net gain, if the hits and false alarms are differentially valued-e.g., if they have different base rates-then $H$ and $F$ should be weighted according to their respective values). Because $M=1-H$ and $V=1-F, M_{\mathrm{p}}=(1-H) /[(1-H)+(1-F)]$. Figure 7 shows $H_{\mathrm{p}}$ and $M_{\mathrm{p}}$ as functions of $c$. Like the findings for net gain, $M_{\mathrm{p}}$ (bottom panel in Figure 7) showed a limited, criterion-specific advantage for small samples (note that for 

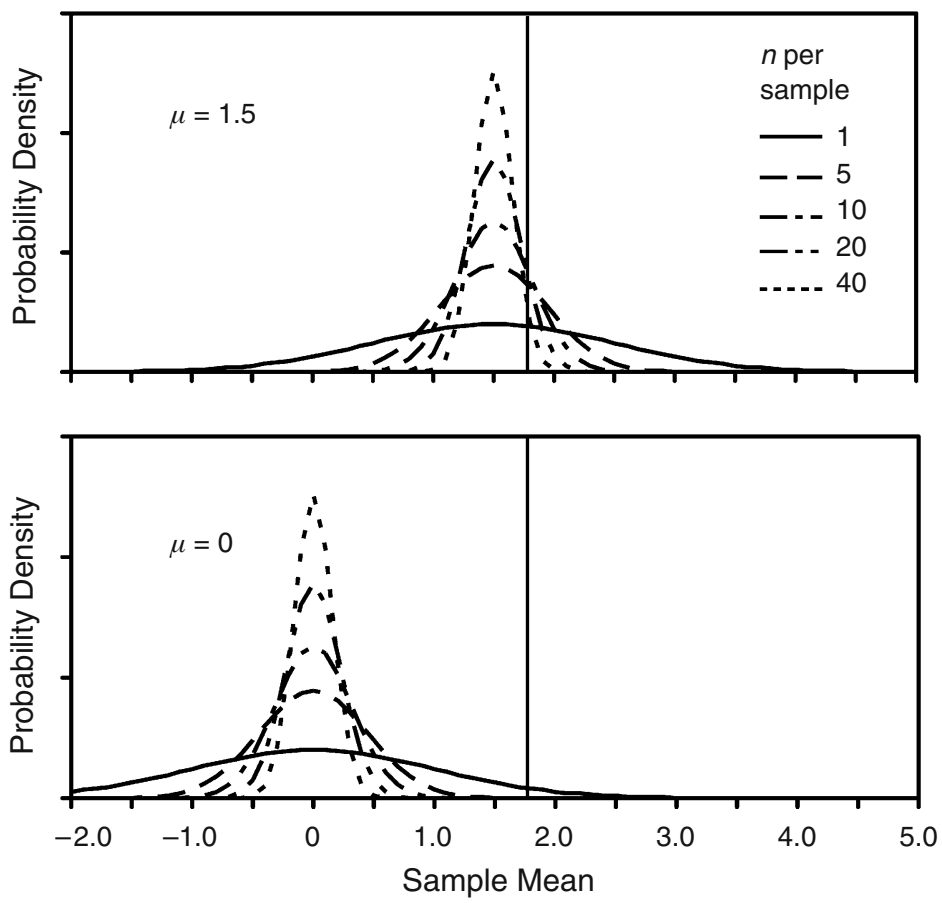

Figure 3. Illustration of the analytic framework for the present study. The curves show theoretical sampling distributions of the mean. The figure shows an arbitrary placement of the decision criterion at 1.75 (denoted by solid vertical lines). Samples falling to the right of the criterion are counted as hits if they are signal samples, and as false alarms if they are noise samples.

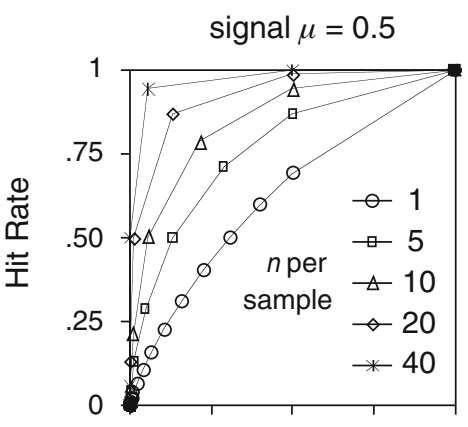

signal $\mu=2$

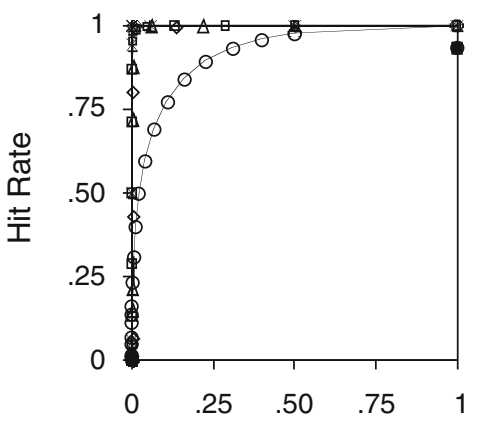

False Alarm Rate

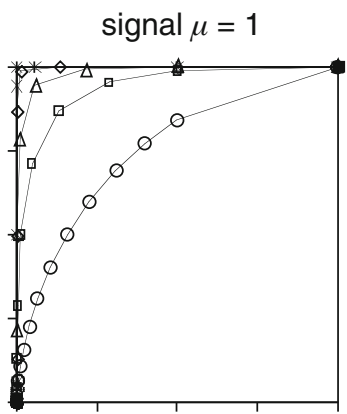

signal $\mu=2.5$

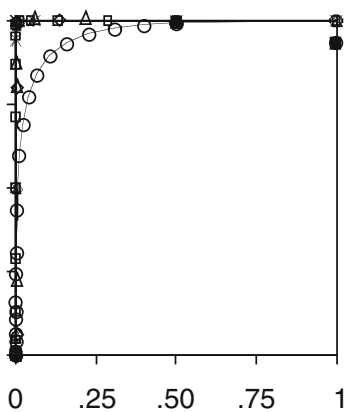

False Alarm Rate signal $\mu=1.5$

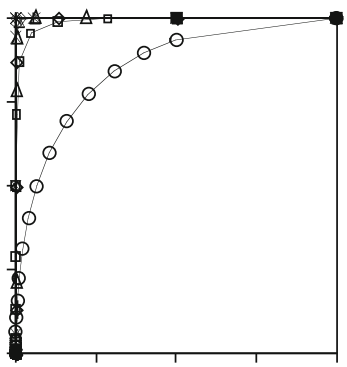

signal $\mu=3$

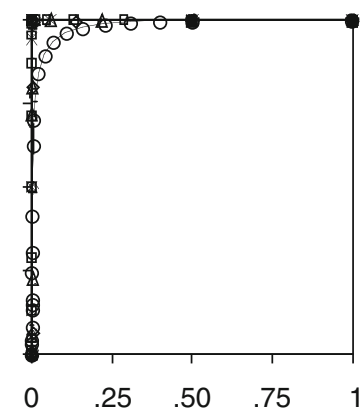

False Alarm Rate

Figure 4. Hit rate $(H)$ as a function of false alarm rate $(F)$ and sample size $(n)$. Each point represents $H$ and $F$ for a particular value of the decision criterion. The area under each curve indicates the discriminability of signal from noise. Note that some curves may be partially obscured because they overlap the edge of the plot area. 


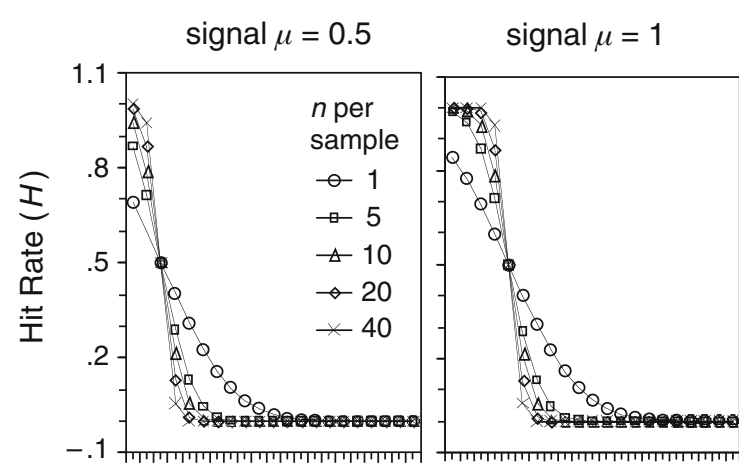

signal $\mu=1.5$
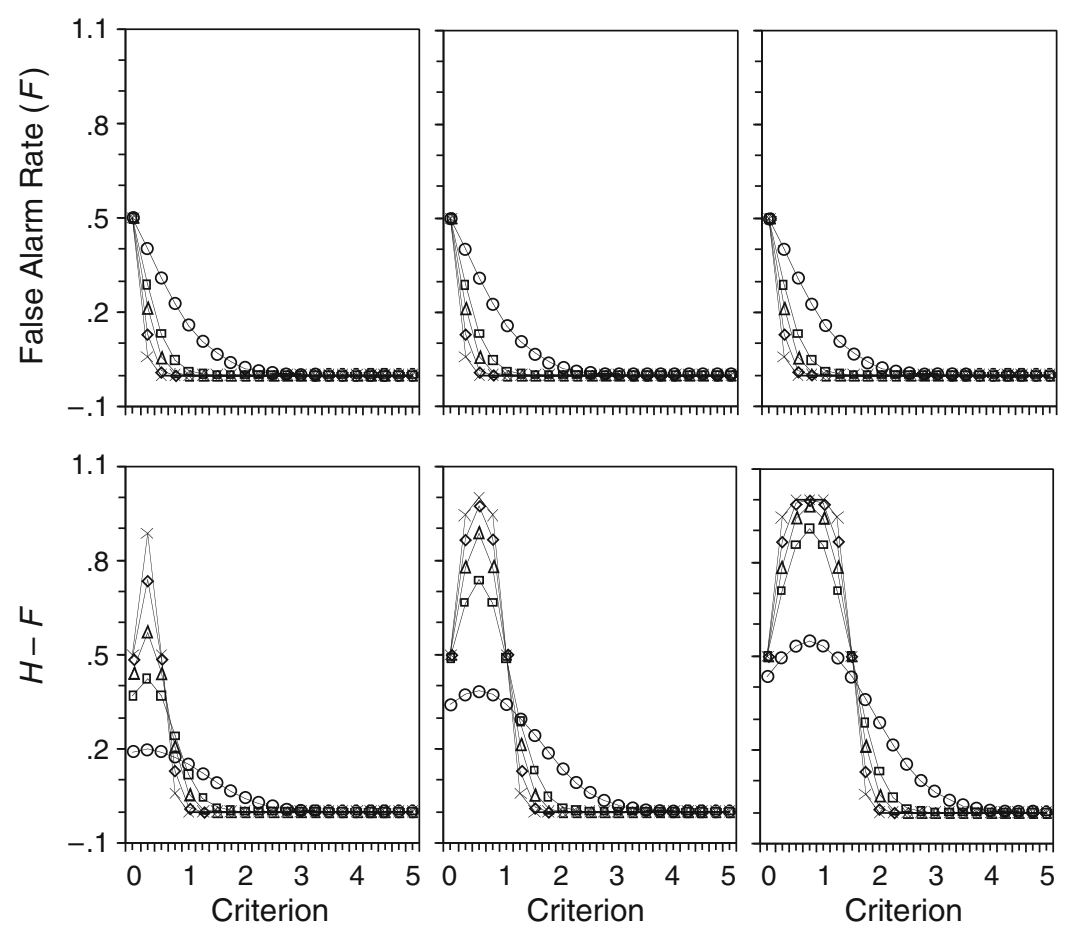

Figure 5. Hit rate $(H)$, false alarm rate $(F)$, and net gain $(H-F)$ as a function of decision criterion and sample size $(n)$ for signal populations with means $(\mu)$ equal to $0.5,1$, and 1.5. In all cases, noise $\mu=0$.

$M_{\mathrm{p}}$, lower values correspond to better performance). When $c$ was low to moderate, the advantage was for large rather than small samples. However, when $c$ became higher than the mean of the signal distribution, the advantage was for small samples, and this advantage became progressively attenuated as $c$ increased further.

In contrast to $M_{\mathrm{p}}$ (and to net gain), the results for $H_{\mathrm{p}}$ (shown in the top panel of Figure 7) never indicated a small-sample advantage. Instead, there was a large sample advantage that was maximal at moderate levels of $c$ and became progressively attenuated as $c$ became extremely high or extremely low.

\section{DISCUSSION}

The results confirm that small-sample advantages can occur in the detection of means, but only when the decision maker uses a suboptimal, conservative decision criterion.
In addition, because sampling distributions of the mean become more variable as $n$ decreases, while remaining normal and centered on the population mean, the present findings demonstrate that sampling variance alone (without sampling skew or central tendency displacement) can produce a small-sample advantage.

\section{Essence of the Small-Sample Advantage}

In the present study, small-sample advantages that occur only with relatively extreme placements of the decision criterion have been identified. Figure 3 illustrates an intuitive explanation for this finding. In the top panel, the areas under the curves to the right of the decision criterion correspond to hits-that is, to the correct classification of signals as signals. False alarms are shown in the bottom panel, again as areas under the curves to the right of the decision criterion. The criterion placement in Figure 3 is such that, when $n$ per sample is large, there are only a few hits and virtually 

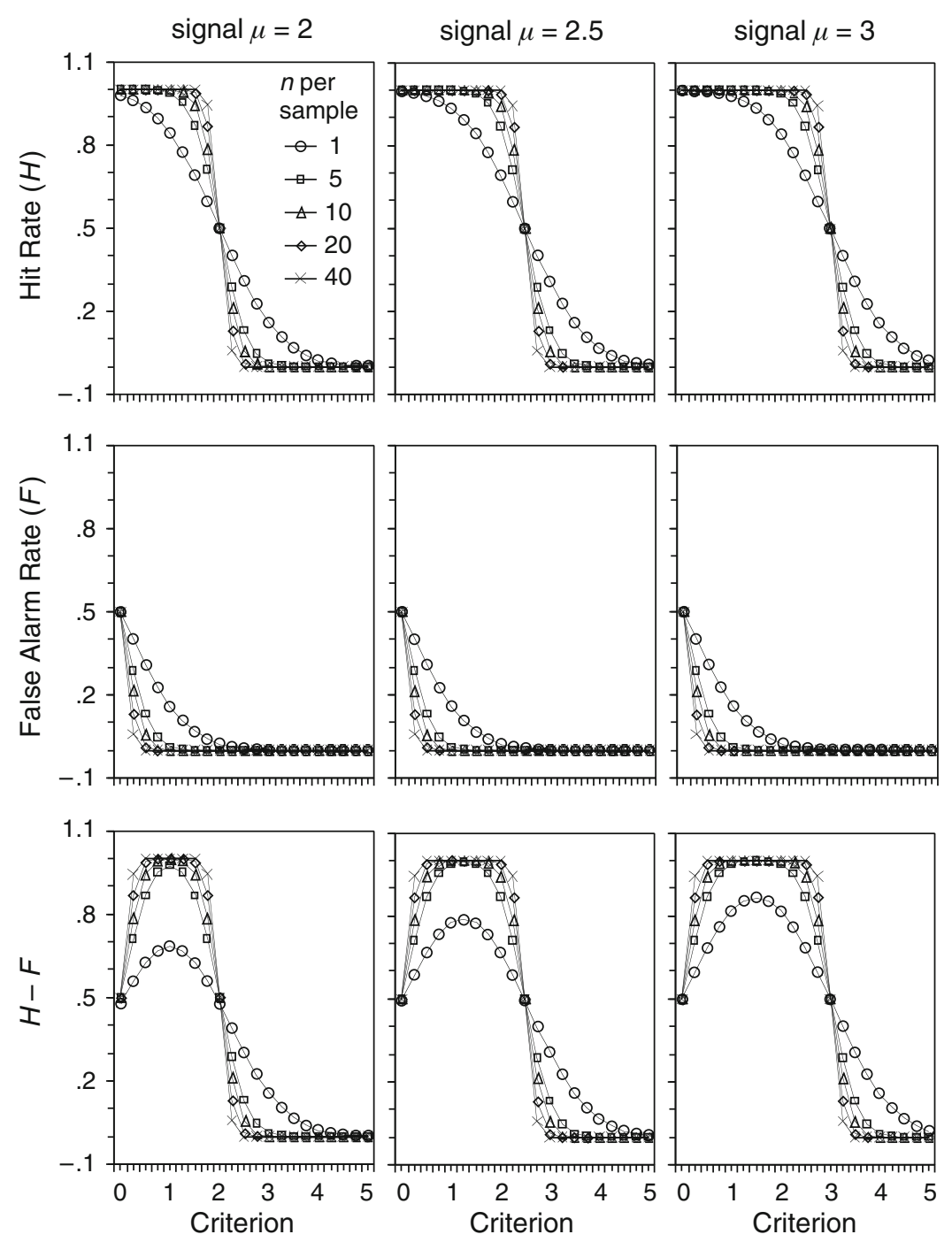

Figure 6. Hit rate $(H)$, false alarm rate $(F)$, and net gain $(H-F)$ as a function of decision criterion and sample size $(n)$ for signal populations with means $(\mu)$ equal to $2,2.5$, and 3 .

no false alarms. But when $n$ is small (e.g., in the $n=1$ condition) there is a large number of hits. At the same time, there are some false alarms, so the increase in hits (as $n$ decreases) is partially offset by an increase in false alarms. However, the number of hits in relation to the number of false alarms is still far greater in the $n=1$ than in the $n=$ 40 condition. Clearly, this effect depends on the criterion's being sufficiently high that under large- $n$ conditions there are few hits and few false alarms, but under small- $n$ conditions there are many hits and relatively few false alarms.

The following behavioral example further illustrates the point. Consider a situation in which an overcautious physician refuses to diagnose the presence of a particular medical condition for which high blood pressure is a key symptom. The overcautious physician withholds judgment unless a patient's average reading (taken on each of several days) is higher than $165 / 105$. If the physician bases each diagnosis on the average of 20 readings, there would likely be very few hits and very few false alarms. For a physician who instead relies on one or two readings per patient, the sampling distributions would be relatively flat and wide, yielding substantial $H$ s and negligible $F$ s, similar to the effect shown in Figure 3. Or consider yet another physician working in a remote, ill-equipped facility, using a blood pressure meter prone to gross random measurement error and attempting to make the kinds of diagnoses described above. In this case, it would be difficult to know whether a particular set of readings is high or low, which might lead the physician to arbitrarily guess where to place criterion. If the guess happens to result in an extremely conservative criterion, then the consequence could be an advantage for small samples, as described above.

\section{Implications for Correlation Detection}

As was noted above, R. B. Anderson et al. (2005) identified sampling variance, skew, and central tendency dis- 

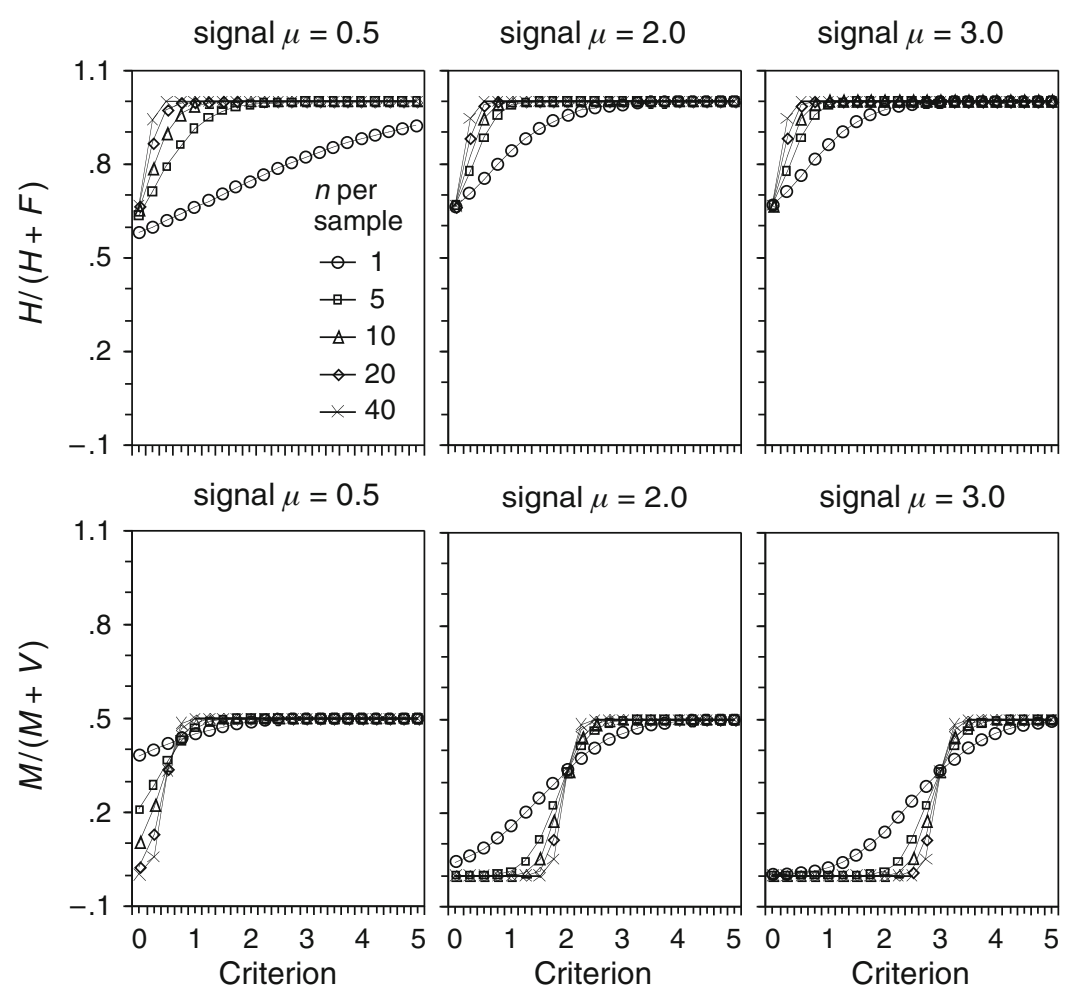

Figure 7. Posterior probability of a hit $\left(H_{\mathrm{p}}\right)$ and posterior probability of a miss $\left(M_{\mathrm{p}}\right)$ as functions of criterion. $M=1-H . V=1-H$. Note that for $M_{\mathrm{p}}$, lower values indicate better performance.

placement as three confounded variables that may give rise to the effect of sample size on correlation detection. The present results for mean detection show that differential sampling variance, as a function of $n$, is a sufficient condition for creating an advantage for small samples. It is therefore likely that differential sampling variance constitutes a sufficient condition for small-sample advantages in correlation detection.

\section{Implications for Adaptive Cognition}

Adaptive criterion placement. The finding of criterion-specific small-sample advantages raises the question of whether it is ever rational for a decision maker to prefer a smaller sample of data over a larger one. One line of reasoning suggests that the answer to this question is "no." In the plots of net gain $(H-F)$ in Figures 5 and 6 , the criterion settings that produce maximal net gain are also the ones that yield an advantage for large samples, not for small ones. Thus, because small-sample advantages occur only under suboptimal criterion conditions, and because rationality would entail optimal criterion placement, it is irrational to prefer a small sample over a large sample. ${ }^{3}$

Net gain versus posterior probability. The results of measuring criterion-specific decision accuracy as $H_{\mathrm{p}}$ or as $M_{\mathrm{p}}$ are shown in the bottom panel of Figure 7. The figure indicates that $H_{\mathrm{p}}$ approaches its maximum as the criterion approaches infinity, leading to the conclusion that the decision maker should always place the criterion as high as possible. At the same time, the results for $M_{\mathrm{p}}$ support an opposite, conflicting strategy: $M_{\mathrm{p}}$ is minimized (and performance is thus maximized) when the criterion is set to the smallest possible value. However, $H_{\mathrm{p}}$ and $M_{\mathrm{p}}$ may be combined to yield the proportion of correct responses. The pattern of results for proportion correct precisely replicates the pattern obtained for net gain. (Proportion correct is simply net gain rescaled.) Thus, the posterior probability analyses and the net gain analyses lead to the same conclusions.

\section{Summary}

The present article demonstrates a limited yet highly counterintuitive phenomenon that runs against the grain of long held convictions that have been bolstered by the practices of traditional null hypothesis testing. But the article may also be understood in terms of a much broader sweep in the psychology of cognition - that is, the increasing attention paid to the necessity of understanding the environment as a prelude to understanding cognitive processes.

\section{AUTHOR NOTE}

This research was supported by Grant SES-0423825 from the National Science Foundation. Correspondence concerning this article should be addressed to R. B. Anderson, Department of Psychology, Bowling Green State University, Bowling Green, OH 43403 (e-mail: randers@bgnet .bgsu.edu). 


\section{REFERENCES}

ANDERSON, J. R. (1990). The adaptive character of thought. Hillsdale, NJ: Erlbaum.

Anderson, R. B., Doherty, M. E., Berg, N. D., \& Friedrich, J. C. (2005). Sample size and the detection of correlation-a signal detection account: Comment on Kareev (2000) and Juslin and Olsson (2005). Psychological Review, 112, 268-279.

Brunswik, E. (1956). Perception and the representative design of psychological experiments (2nd ed.). Berkeley: University of California Press.

BRUnSWIK, E., \& KAMIYA, J. (1953). Ecological cue-validity of "proximity" and of other Gestalt factors. American Journal of Psychology, 66, 20-32.

DAVID, F. N. (1954). Tables of the ordinates and probability integral of the distribution of the correlation coefficient in small samples. Cambridge: University Press.

Doherty, M. E. (2001). Demonstrations for Gestalt psychologists: Psychology without a subject. In K. R. Hammond \& T. R. Stewart (Eds.), The essential Brunswik: Beginnings, explications, applications (pp. 211-213). New York: Oxford University Press.

FiedLer, K., \& KareEv, Y. (2006). Does decision quality (always) increase with the size of information samples? Some vicissitudes in applying the law of large numbers. Journal of Experimental Psychology: Learning, Memory, \& Cognition, 32, 883-903.

GigerenZER, G., \& ToDD, P. M. (1999). Fast and frugal heuristics: The adaptive toolbox. In G. Gigerenzer, P. M. Todd, \& The ABC Research Group (Eds.), Simple heuristics that make us smart (pp. 3-34). New York: Oxford University Press.

Juslin, P., \& Olsson, H. (2005). Capacity limitations and the detection of correlations: Comment on Kareev (2000). Psychological Review, 112, 256-267.

KAREEV, Y. (1995). Through a narrow window: Working memory capacity and the detection of covariation. Cognition, 56, 263-269.

Kareev, Y. (2000). Seven (indeed, plus or minus two) and the detection of correlations. Psychological Review, 107, 397-402.
Kareev, Y., Lieberman, I., \& Lev, M. (1997). Through a narrow window: Sample size and the perception of correlation. Journal of Experimental Psychology: General, 126, 278-287.

Macmillan, N. A., \& Creelman, C. D. (1991). Detection theory: A user's guide. New York: Cambridge University Press.

SHEPARD, R. N. (1990). Mind sights: Original visual illusions, ambiguities, and other anomalies, with a commentary on the play of mind in perception and art. New York: Freeman.

Simon, H. A. (1969). The sciences of the artificial. Cambridge, MA: MIT Press.

Stanislaw, H., \& Todorov, N. (1999). Calculation of signal detection theory measures. Behavior Research Methods, Instruments, \& Computers, 31, 137-149.

YoNELINAS, A. P. (2002). The nature of recollection and familiarity: A review of 30 years of research. Journal of Memory \& Language, 46, 441-517.

\section{NOTES}

1. Criterion-specific accuracy can also be measured as the hit rate $(H)$ divided by the sum of $H$ and the false alarm rate $(F)$, or as the miss rate divided by the sum of the miss rate $(1-H)$ and the correct rejection rate $(1-F)$ (see R. B. Anderson, Doherty, Berg, \& Friedrich, 2005; Juslin \& Olsson, 2005).

2 . The present examples assume that the raw data are normally distributed. Therefore, the mean, median, and mode are identical.

3. In a set of unpublished simulations, the authors manipulated the value of hits relative to the negative value of false alarms and calculated net gain as the value-weighted $H$ minus the value-weighted $F$. The manipulation did not span all possible weightings. However, the authors were unable to find a set of weights that produced a small-sample advantage under conditions that also maximized net gain.

(Manuscript received February 10, 2005; revision accepted for publication August 24, 2005.) 